INDAGINE CONOSCITIVA SULL'UTILIZZO

FRAUDOLENTO DI SOSTANZE ANABOLIZZANTI NEI BOVINI MACELLATI IN MOLISE

\title{
A SURVEY ON THE FRAUDULENT USE OF ANABOLIC SUBSTANCES IN BOVINES SLAUGHTERED IN MOLISE
}

Imbimbo P. ${ }^{1}$, Castigliego L. ${ }^{1}$, Armani A. ${ }^{1}$, Di Zinno A. ${ }^{2}$, Niro A. ${ }^{2}$, Guidi A., Gianfaldoni D. ${ }^{1}$

${ }^{1}$ Dipartimento di Patologia Animale, Profilassi ed Igiene degli Alimenti - Università di Pisa

${ }^{2}$ Azienda Sanitaria Regione Molise

SUMMARY

KEYWORDS

\begin{abstract}
An investigation has been performed on the fraudulent use of anabolic substances in the Region of Molise. One hundred fourty-four bovines (12-24 months old, 123 males and 21 females) have been included in the survey. Antemortem assessment on their behaviour and clinical analysis on some target organs were carried out. After slaughtering, samples of prostate, bulbo-urethral glands, Bartholin's glands, mammary gland, ovaries, thymus and thyroid were collected and processed for an anatomo-histopathological evaluation, as suggested in the guidelines of the Italian National Plan for Residues (PNR) 2009. Overall, the $15 \%$ of the subjects analysed have been classified as "suspect", whereas the $44 \%$ as "uncertain" and the remaining $59 \%$ as "negative". The lesion most frequently found was a serious atrophy of the thymic parenchyma with fat infiltration (15\% of males and $14 \%$ of females), suggesting a prevalence of an illegal use of cortisonic drugs.
\end{abstract}

anabolic substances, illegal drug treatments, bovines

\section{INTRODUZIONE}

Sin dagli anni ' 60 , in Italia, è stata vietata la somministrazione di sostanze anabolizzanti a scopo auxinico negli animali destinati all'alimentazione umana. Da allora si sono succeduti aggiornamenti legislativi che hanno portato al D.L. 148/2009, attuazione della Direttiva 2008/97/CE (2), che modifica il D.L.158/2006 (3) e successive modificazioni, concernente il divieto di utilizzazione di talune sostanze ad azione ormonica, tireostatica e $\beta$-agoniste nelle produzioni animali.

In particolare, permangono i divieti assoluti di somministrazione, "mediante qualsiasi metodo, agli animali d'azienda e agli animali d'acquacoltura, di tireostatici, stilbeni e derivati dello stilbene e loro sali ed esteri, estradiolo-17 beta e suoi derivati sotto forma di esteri e sostanze beta-agoniste e di sostanze ad azione estrogena diverse dall'estradiolo-17 beta e dai suoi derivati sotto forma di esteri - androgena o gestagena, nonché qualsiasi altra sostanza ad effetto anabolizzante", salvo in caso di trattamenti terapeutici o zootecnici regolamentati. A tal proposito, nel D.L. 158/2006 si chiarisce il concetto di "trattamento illecito", definendolo come "utilizzazione di sostanze o prodotti non autorizzati, ovvero di sostanze o prodotti autorizzati a fini e condizioni diverse da quelli previsti dalle disposizioni vigenti."

Infatti, gli studi effettuati sino ad oggi tendono a concordare sul fatto che un'eccessiva esposizione agli ormoni sessuali o a residui di altre sostanze anabolizzanti possa determinare effetti nocivi su ghiandole endocrine, alterando crescita e pubertà, sistema immunitario, oltre ad esercitare effetti genotossici e carcinogenetici (4).

Ciò nonostante, a dispetto di una rigida legislazione e di un piano nazionale residui(PNR) per la ricerca negli alimenti che impone precise regole circa il numero e la modalità di campionamento da effettuare, il problema del trattamen- 
to illecito con ormoni promotori della crescita è ancora di scottante attualità. L'utilizzo di sostanze anabolizzanti è favorito dal fatto che esse non possono essere rilevate facilmente, sia per la loro bassa concentrazione nei campioni biologici, sia per il numero molto elevato delle molecole potenzialmente utilizzabili, spesso modificate rispetto a quelle indicate nei protocolli ufficiali, sia per l'impiego dei cosiddetti cocktail: miscele di molecole che, agendo sinergicamente, consentono di ottenere ottimi risultati zootecnici, abbassando la concentrazione relativa di ogni singolo composto utilizzato e, quindi, anche quella di eventuali residui, comportando ulteriori difficoltà dal punto di vista analitico.

Tuttavia, tali sostanze, utilizzate nelle produzioni animali, causano la comparsa di alterazioni più $o$ meno evidenti in altri organi che rispondono al loro effetto stimolante o inibente (5). Infatti, nel corso degli anni, studi in proposito hanno suggerito come l'utilizzo di composti anabolizzanti possa essere messo in evidenza ricercando alterazioni sia a livello macroscopico sia a livello istologico (6) su alcune componenti dell'apparato genitale maschile o femminile ( 7 , $8,9)$, sulle ghiandole sessuali accessorie $(10,11)$ o su alcuni organi particolarmente responsivi ad alcune tipologie di trattamento, come il timo $(12,13)$ o la tiroide (14). Tali lesioni perdurano per un tempo sufficientemente lungo da poter essere rilevate al momento della macellazione.

Infatti, oltre alle classiche analisi chimiche - le uniche, comunque, che hanno valore legale- , i mezzi di ausilio diagnostico per evidenziare un trattamento con steroidi sessuali e molecole ad attività simile si basano su osservazioni cliniche e di eventuali modificazioni comportamentali e su analisi anatomo-isto-patologiche di alcuni organi bersaglio (6), la cui valutazione mediante test di screening è stata ufficialmente introdotta nel PNR 2008. Limitandoci comunque al bovino, esula da questo contesto esplicitare in dettaglio i parametri relativi alle alterazioni del comportamento e morfo-funzionali a carico di alcuni organi coinvolti o le modificazioni relative ai parametri zootecnici caratteristiche della razza. Ci limiteremo, pertanto, ad elencare brevemente nella sezione dedicata ai materiali e metodi le alterazioni anatomo-isto-patologiche ricercate, relative ai gruppi di sostanze scelte per effettuare il presente studio (androgeni, estrogeni, cortisonici, tireostatici).

Nell'anno 2009, l'Azienda Sanitaria Regione Molise, in collaborazione con il Dipartimento di Patologia Animale, Profilassi ed Ispezione degli Alimenti dell'università di Pisa hanno organizzato uno studio dal titolo: "Applicazione del test istologico nella prevenzione dell'uso di anaboliz- zanti in zootecnia". Il fine è stato quello di ottenere dati epidemiologici sull'uso di sostanze anabolizzanti in bovini macellati nella Regione Molise e di comprendere, quindi, le dimensioni e l'estensione del fenomeno.

\section{MATERIALI E METODI}

Campione esaminato: La popolazione oggetto di studio è rappresentata da bovini di età compresa tra i 12 e i 24 mesi, macellati in Molise nel 2009, ma provenienti anche dalla regione Campania (34\%). Sulla base dei dati produttivi locali, riferiti al volume delle macellazioni, è stato estrapolato un campione statisticamente rappresentativo in termini di partite da sottoporre a verifica. Poiché tra gli obiettivi del progetto è prevista la valutazione del livello di sicurezza delle carni provenienti da bovini macellati in Molise, è stato deciso che la procedura del calcolo delle partite da campionare dovesse tener conto di una prevalenza attesa massima del $2 \%$.

$\mathrm{Su}$ una popolazione di 11.000 bovini macellati/anno, raggruppati in circa 8000 partite, e considerando un livello di confidenza del $95 \%$, è risultato che si sarebbero dovute campionare 147 partite. Le partite erano mediamente costituite da 1-2 soggetti. In questa indagine, è stato possibile esaminare 144 soggetti, di cui 123 vitelloni e 21 scottone.

Per ogni capo è stato effettuato il controllo documentale comprensivo di:

- - numero, età e razza dell'animale;

- - ubicazione dell'allevamento di provenienza;

- - dichiarazione dell'allevatore che l'animale non avesse subito trattamenti farmacologici negli ultimi 90 giorni.

Ogni capo è stato sottoposto a regolare visita ante-mortem, ricercando, inoltre, alterazioni morfo-funzionali di alcuni organi ispezionabili alla visita clinica.

In particolare, per la valutazione del trattamento con estrogeni:

- $\quad$ - maschi: dimensione dei testicoli e sviluppo della ghiandola mammaria;

- - femmine: dimensione e sviluppo della ghiandola mammaria e dei capezzoli.

Per la valutazione del trattamento con androgeni:

- - maschi: dimensione dei testicoli;

- - femmine: dimensione del clitoride.

Per la valutazione del trattamento con corticosterodi si è proceduto al prelievo del timo, dopo macellazione, per la misurazione del volume e del peso.

Prelievo campioni ed analisi istologica: per l'analisi istologica sono stati prelevati prostata, ghiandole bulbo uretrali, ghiandola di Bartolino, ghiandola mammaria, ovaie, timo e tiroide, 
a seconda del sesso. Il materiale biologico è stato fissato in soluzione di formalina al $4 \%$. I campioni sono stati numerati in maniera progressiva. Il campione è stato successivamente processato secondo il seguente schema riassuntivo:

- $\quad$-riduzione del pezzo anatomico (trimming);

- -disidratazione in alcol ascendenti e diafanizzato in xilolo;

- -inclusione in paraffina;

- -realizzazione delle sezioni istologiche con microtomo;

- $\quad$ colorazione della sezione con ematossilinaeosina;

- -montaggio del vetrino;

- $\quad$-lettura e diagnosi.

La valutazione diagnostica, realizzata in collaborazione con il Dipartimento di Patologia Animale dell'Università degli Studi di Torino, è stata effettuata tramite l'analisi visiva del singolo organo per la ricerca delle eventuali lesioni, come indicato sul PNR 2009. In seguito, sono state compilate le relative schede di valutazione.

Il singolo organo è stato quindi classificato come "negativo", quando le caratteristiche dei diversi tessuti fossero proprie degli animali di controllo, cioè fisiologiche; "sospetto", quando anche uno solo dei tessuti esaminati presentasse lesioni gravi e note in letteratura, chiaramente riferibili a trattamenti illeciti, oppure, come indicato dalla scheda di valutazione, quando si fosse verificata l'associazione di due parametri dubbi; "dubbio" qualora il campione presentasse lesioni lievi oppure di nuova comparsa, non fisiologiche, ma non ancora descritte in letteratura.

Tabella 1.
Per quanto riguarda la classificazione del soggetto di cui si sono esaminati gli organi bersaglio, "negativo" si riferisce ad una condizione per la quale tutti gli organi siano stati classificati come negativi; "sospetto", nel caso in cui anche un solo organo sia stato classificato come tale; "dubbio", se anche un solo organo sia stato classificato dubbio, ma nessuno come sospetto.

\section{RISULTATI}

Non è stata rilevata alcuna alterazione macroscopica relativa ai testicoli o alla ghiandola mammaria dei soggetti esaminati.

Sull'intera popolazione campionata, il 15\% dei soggetti è stata classificata come "sospetto", mentre il $44 \%$ come "dubbio" ed il restante $59 \%$ come "negativo".

Le conoscenze attuali non ci hanno permesso di attribuire, con adeguato grado di certezza, la genesi delle lesioni di grado lieve ad un trattamento con ormoni promotori della crescita. Resta di fatto che anche i soggetti risultati dubbi erano animali che presentavano un quadro istologico sicuramente non fisiologico.

Analizzando i risultati distintamente tra i due sessi, possiamo osservare come le percentuali di sospetti, dubbi e negativi siano pressoché identiche, nonostante il numero delle scottone sottoposte a prelievo fosse molto inferiore a quello dei vitelloni,

Per quanto riguarda i risultati relativi ai singoli organi, rimandiamo alla tabella di seguito riportata, che indica il numero di soggetti classificati in base ai criteri precedentemente descritti. In parentesi le relative percentuali sul totale degli organi analizzati:

\begin{tabular}{|c|c|c|c|c|c|}
\hline & NEGATIVO & DUBBIO & SOSPETTO & N.P. & TOTALE \\
\hline PROSTATA & $82(68 \%)$ & $38(31 \%)$ & $1(1 \%)$ & 2 & 123 \\
\hline Gh. BULBO-URETRALE & $112(92 \%)$ & $10(8 \%)$ & $0(0 \%)$ & 1 & 123 \\
\hline TIMO & $86(62 \%)$ & $31(23 \%)$ & $21(15 \%)$ & 6 & 144 \\
\hline Gh. BARTOLINO & $8(47 \%)$ & $9(51 \%)$ & $0(0 \%)$ & 4 & 21 \\
\hline MAMMELLA & $13(100 \%)$ & $0(0 \%)$ & $0(0 \%)$ & 8 & 21 \\
\hline OVAIE & $8(100 \%)$ & $0(0 \%)$ & $0(0 \%)$ & 13 & 21 \\
\hline TIROIDE & $140(98 \%)$ & $3(2 \%)$ & $0(0 \%)$ & 1 & 144 \\
\hline
\end{tabular}

N.P. : non analizzati

L'organo in cui è stato riscontrato il maggior numero di alterazioni, potenzialmente imputa- bili a trattamenti con sostanze promotrici di crescita, è il timo. Sia nei maschi che nelle 
femmine, infatti, la percentuale di soggetti che hanno riportato quadri istologici di grave atrofia del parenchima timico è elevata e risulta rispettivamente pari al $15 \%$ e al $14 \%$ per un totale di 22 soggetti .

In figura è riportato un esempio delle alterazioni riscontrate a livello del timo. In particolare, il campione istologico proviene da un maschio di circa 17 mesi che alla lettura del preparato istologico riportava un quadro di atrofia del parenchima timico con deplezione linfocitaria e graduale deposito di tessuto adiposo. Il fenomeno si diffonde dalla periferia della corticale in direzione della midollare.

\section{Figura 1.}

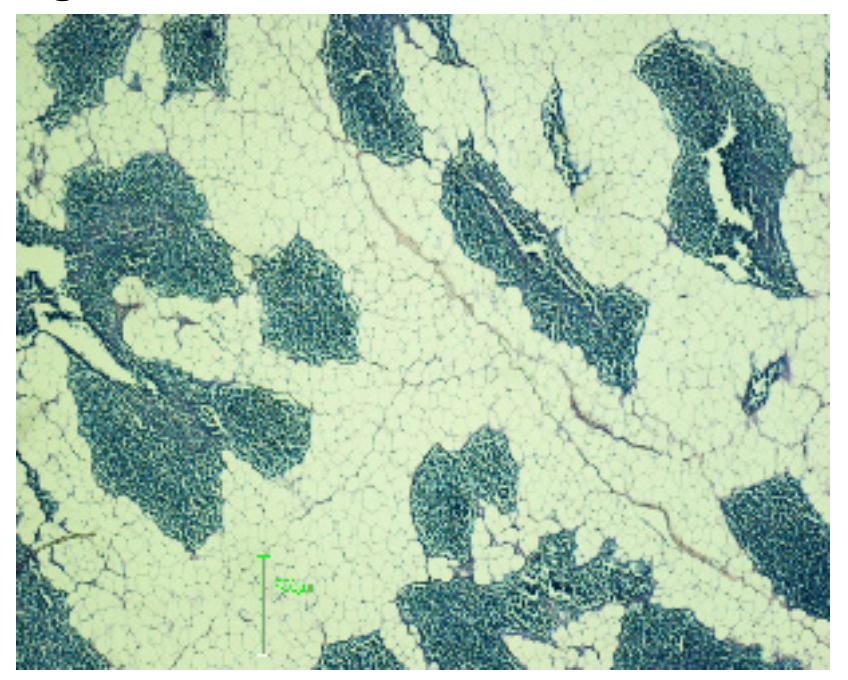

\section{CONSIDERAZIONI E CONCLUSIONI}

La maggior parte delle lesioni riscontrate ha riguardato il timo. Questa evidenza, associata all'assenza di un'eventuale denuncia di trattamento farmacologico terapeutico, diventa un forte indicatore di probabile trattamento illecito con cortisonici a scopo fraudolento. Ricordiamo che l'uso dei cortisonici, oltre ad avere notevoli effetti positivi sulla resa al macello, provocando un aumento delle massa dovuto a ritenzione idrica e aumento dei liquidi extracellulari, determina un'atrofia più o meno marcata del timo, con deplezione linfocitaria e graduale deposito di tessuto adiposo (15). E' inoltre dimostrato che l'atrofia timica nei giovani soggetti (vitelli e vitelloni) sia reversibile nell'arco di circa due settimane da un regolare trattamento con corticosteroidi (16).

I risultati di questa indagine suggeriscono, quindi, un'elevata probabilità che l'impiego di sostanze anabolizzanti vietate dalla legge sia pratica a tutt'oggi diffusa sul territorio. In particolare, i dati ottenuti da questo studio indicano percentuali di individui sospetti tutt'altro che esigue, rivelando una portata del fenomeno che, se dovesse essere confermata, risulterebbe di proporzioni preoccupanti e del tutto in contrasto con i dati ottenuti annualmente dal PNR, in cui si rilevano positività mediamente inferiori al punto percentuale.

Nei casi in cui, ad un'indagine istologica, siano riscontrate evidenti alterazioni degli organi, la legislazione vigente fornisce precise indicazioni circa le procedure da attuare negli allevamenti di provenienza dei soggetti risultati sospetti di trattamento illecito. Come ampiamente precisato nel PNR, ricordiamo che i piani di monitoraggio basati sul test istologico rappresentano un elemento integrativo e non sostitutivo del controllo chimico, che resta attualmente l'unico ad avere validità giuridico-legale. Tutto ciò si traduce nell'obbligo di identificare inequivocabilmente, nei campioni biologici dell'animale, la molecola responsabile dell'alterazione, prima di poter esprimere un parere legalmente valido di positività del soggetto. Tuttavia, se alcune alterazioni istologiche permangono fino al momento della macellazione, le metodiche analitiche utilizzabili ad oggi possono non essere sufficientemente sensibili per la conferma della positività.

\section{BIBLIOGRAFIA}

1. Decreto Legislativo n.148 del 29 ottobre 2009. Gazzetta Ufficiale n. 254 del 31 ottobre 2009 .

2. Direttiva del Parlamento Europeo e del Consiglio n. 97, del 19 novembre 2008. Gazzetta Ufficiale n. 318 del 28 novembre 2011..

3. Decreto Legislativo n. 158 del 6 marzo 2006. Gazzetta Ufficiale n.98 del 28 aprile 2006.

4. European Food Safety Authority (2007). Opinion of the scientific panel on contaminants in the food chain on a request from the european commission related to hormone residues in bovine meat and meat products Question N ${ }^{\circ}$ EFSA-Q-2005-048. The EFSA Journal, 510, 1-62.

5. Biolatti, B., Bollo, E., Appino, S. (1993). Patologia dell'apparato genitale, apparato respiratorio, mammella e timo di vitelli trattati con clenbuterolo e steroidi sessuali. Argomenti di Patologia Veterinaria, 285-302.

6. Biolatti, B., Cabassi, E., Rosmini, R. (2003). Lo screening istologico nella prevenzione dell'uso di anabolizzanti nel bovino. Large Animal Review, 9, 9-19.

7. Jansen, E.H., Stepany, R.W., Vos, J.G., Ruitenberg, E.J., Benraad, Th.J., De Boer, F., De Ruig, W.G., Weijman, J., Schmidt, N.A. (1989). Application of diethylstilbestrol dipropionate in bulls. Excretion of residues in urines and faeces and histological and 
immunoistochemical chenges in the prostate. The Veterinary Quarterly, 11, 1-11.

8. Groot, M., den Hartog, J.M., Gruys, E. (1989). Influence of androgens on the genital tract of cyclic heifers. The veterinary Quarterly, 11,198-209.

9. Groot, M., Den Hartog, J.M.P. (1990). Histological changes in the genital tract of female veal calves implanted with naturally occurring anabolic steroids. Journal of Veterinary Medicine Series A, 45, 425-440.

10. Rosmini, R., Marcato, P.S., Marocchio, L. (1987). Aggiornamento interpretativo dei reperti istologici nella prostata e nella ghiandola di bartolino di vitelli macellati. Praxis Veterinaria, 8, 8-10.

11. Corradi, A., Cantoni, A.M, Borghetti, P., Cabassi, E. (1990). Risposte istochimiche delle ghiandole annesse all'apparato genitale in vitelli trattati con anabolizzanti. Atti della società italiana di buiatria. 12, 639649.

12. Guarda, F., Valenza, F., Biolatti, B., Quaglia, F., Emanuel, C. (1984). Sull'atrofia precoce cel timo in seguito a somministrazione prolungata di glicocorticoidi nei vitelli a carne bianca regolarmente macellati. Il nuovo progresso veterinario $\mathrm{n}^{\circ} 9$.

13. Guarda, F., Biolatti, B., Valenza, F., Miglietti, M. (1990). Correlazioni anatomopatologiche tra lesioni del timo e dell'apparato genitale femminile di vitelli a carne bianca regolarmente macellati. Deutsch TierArztl Wschr, 79, 313-315.

14. Seren, E., Mora, A. (1973). Funzionalità tiroidea di bovini normali e trattati con metiltiouracile (possibilità di riconoscere in vivo gli animali trattati con antitiroidei. Folia Veterinaria Latina, 3, 52-73.

15. Guarda, F, Valenza, F., Biolatti, B., Quaglia, F., Emanuel, C. (1984). Sull'atrofia precoce del timo in seguito a somministrazione prolungata di glicocorticoidi nei vitelli sanati. Il Nuovo Progresso Veterinario, 9, 39.

16. Biolatti, B., Cannizzo, T., Zancanaro, G., Amedeo, S., Cesano, L., Barbarino, G. (2003). Effects of low doses of dexamethasone on bovine thymus morphology. Research in Veterinary Science, 74 (suppl. A), 12. 